Western University

Scholarship@Western

Physics and Astronomy Publications

Physics and Astronomy Department

2013

Influence of Electrostatic Forces on the Particle Propulsion in the Evanescent Field of Silver IonExchanged Waveguides

Dmytro Gebennikov

Western University

Silvia Mittler

Western University, smittler@uwo.ca

Follow this and additional works at: https://ir.lib.uwo.ca/physicspub

Part of the Astrophysics and Astronomy Commons, and the Physics Commons

Citation of this paper:

Gebennikov, Dmytro and Mittler, Silvia, "Influence of Electrostatic Forces on the Particle Propulsion in the Evanescent Field of Silver Ion-Exchanged Waveguides" (2013). Physics and Astronomy Publications. 46.

https://ir.lib.uwo.ca/physicspub/46 
Langmuir 29 (2013) 2615-2622

\title{
Influence of Electrostatic Forces on the Particle Propulsion in the Evanescent Field of Silver Ion-Exchanged Waveguides
}

\author{
Dmytro Gebennikov and Silvia Mittler \\ Department of Physics and Astronomy, The University of Western Ontario (Western \\ University), London, Ontario, N6A 3K7, Canada
}

\begin{abstract}
The effect of electrostatic interaction between carboxylate- and amino-functionalized polystyrene particles and a charged waveguide surface on the propulsion speed in an optical tweezers is considered as a function of $\mathrm{pH}$ and ionic strength. It was shown that with the variation of the $\mathrm{pH}$ of the aqueous solution, the particles were immersed in, a systematic change of propulsion speed with a maximum speed could be achieved. The appearance of a maximum speed was ascribed to changes in the particle-waveguide separation as a result of the combination of two forces: Coulomb repulsion/attraction and induced dipole forces. The highest maximum speed at low ionic strength was around $12 \mu \mathrm{m} / \mathrm{sec}$. Changes in the ionic strength of the solution influenced the gradient of the dielectric constant near the involved surfaces and also lead to a slightly reduced hydrodynamic radius of the particles. The combination of these effects subsequently increased the maximum speed to about $23 \mu \mathrm{m} / \mathrm{sec}$.
\end{abstract}




\section{Introduction}

The transition from large scale systems for particle and biological cell analysis to the socalled lab-on-a-chip systems not only allows decrease in the amount of necessary analyte, and subsequently reduced cost, but also increases the speed, the availability and the accuracy of the analysis. Microfluidic-based systems offer the possibility to integrate several functional modules for sample transport, sorting and, finally, analysis on a single chip [1]. Different mechanical, electrical and magnetic based technologies, each having their advantages and drawbacks, have been suggested for sample transport and sorting [2].

Optically based methods that emerged after the first demonstration of optical trapping of microspheres by a laser beam by Ashkin [3] opened an alternative for particle manipulation. Traditional optical tweezers take advantage of focused laser beams to trap and steer single microparticles in three dimensions [4]. As a modification of a single particle trap, Curtis et al. [5] demonstrated trapping and aligning of a large number of particles. High spatial resolution and high trapping forces enabled trapping and manipulating of object ranging in size from several nanometers to microns $[6,7,8]$. Optical tweezers found applications in such areas as biology [9], medicine [10], micromechanics [11] and statistical mechanics [12].

By using the entire power available in a laser beam for trapping and manipulating objects, the classical optical tweezers can be damaging and thus are unsuitable for some biological cells. Waveguide-based techniques for trapping and manipulating of micro-particles offer the possibility for particle manipulation in two dimensions, but the guided wave optical tweezers rely on the interaction of the objects with the evanescent field. Using near-field trapping allows the application of various integrated optical structures, such as dividers and couplers. In waveguide-based optical 
tweezers the optical gradient force pulls the particles towards the surface of the waveguide, whereas the radiation force propels them in the direction of light propagation [13]. The first demonstration of trapping a micro-particle by an evanescent field on the surface of a prism was carried out by Kawata and Sugiura [14] followed by an experiment on particles propulsion in the evanescent field of a channel waveguide by Kawata and Tani [15]. Trapping and propulsion of gold nanoparticles [16-18], polystyrene particles with dimensions in micrometer range [19-23] as well as nanowires [24] has been reported on channel waveguides. The first trapping and propulsion of live cells in the evanescent field of waveguide was demonstrated by Gaugiran et al. [25] with red blood cells and yeast cells. However, the application of near-field systems on live cells bears several issues: e.g. small differences in the refractive index between cells and the surrounding medium (water), which determines the optical forces and the adhesion of the cells to each other and to the surface of the waveguide [26,27]. Ahluwalia et al. [28] studied the propulsion of nonadherent red blood cells, however observed that they stuck to the surface of the waveguide. Authors assumed the presence of electrostatic forces responsible for the inhibition of the cell propulsion.

In this publication we considered a controlled electrostatic interaction between dielectric micro-spheres and the surface of a silver ion-exchanged channel waveguide containing chargeable groups: a model system for studying propulsion of particles with Coulomb interaction. We implemented particles containing amino- or carboxylate- functional groups at their surfaces as well as waveguide surfaces with amino groups. The $\mathrm{pKa}$ value of the $-\mathrm{NH}_{2}$ group is around 9 , while that of $-\mathrm{COOH}$ lies around 2 [29] of cause depending individually on the precise molecular structure. Thus, variation of the $\mathrm{pH}$ of the aqueous environment of both particles and waveguide surface should allow the creation of positive and negative charges on the surfaces of particles and 
waveguide and, subsequently, appearance of attractive and repulsive forces between the two surfaces, respectively. It was assumed that the electrical double layer will play a significant role; therefore, the ionic strength of the environmental solution was controlled as well.

\section{Experimental Section}

Waveguide Fabrication. Waveguide samples containing $10 \mu \mathrm{m}$ wide channels were fabricated in BK-7 glass (Hellma Optics, Germany) through a silver ion exchange process [30]. Standard photolithographic procedures were employed. After a thorough cleaning process to remove all residuals, the surface of the glass was spin coated (Solitec 5110 Spinner/Developer, USA) with $600 \mathrm{~nm}$ of S1805 photoresist (Shipley, USA). With the help of a mask aligner (Karl Süss MA6 Aligner, Germany) the $10 \mu \mathrm{m}$ wide channels were patterned and developed for $40 \mathrm{sec}$ in MF319 developer (Shipley, USA). A $100 \mathrm{~nm}$ thick aluminium layer was then deposited in a custom made e-beam evaporation system (Nortel, Canada) followed by a lift off procedure. This lead to an aluminium mask with open channels on top of the glass sample. Silver ion exchange was carried out at $360 \mathrm{C}^{\circ}$ in a binary mixture of silver nitrate and sodium nitrate $(99.0 \%$, Caledon Laboratories Ltd., Canada). High absorption losses in silver ion exchanged waveguides observed previously [31] are caused by light scattering and absorption from silver clusters formed during the ion exchange process. In order to reduce these losses a series of slab waveguides in melts consisting of binary mixtures of silver and sodium nitrates with different concentrations (molar percent of silver nitrate varied from 1 to $10 \%$ ) were prepared. Waveguides were characterized by m-line spectroscopy [32]. It was found that the refractive index change at the surface of the slab ion-exchanged waveguide $(\Delta \mathrm{n})$ increased from 0.04 for $1 \% \mathrm{AgNO}_{3}$ to 0.07 for $5 \%$ of $\mathrm{AgNO}_{3}$.

Further increase in the amount of $\mathrm{AgNO}_{3}$ did not change $\Delta \mathrm{n}$ but lead to high losses. Based on this 
knowledge, all channel waveguides were fabricated in a $5 \% \mathrm{AgNO}_{3}$ melt. Walker et al. [33] studying silver ion-exchanged waveguides observed the formation of metallic silver along the sides of channel waveguides during ion exchange. They assumed the reason for this metallic silver formation is due to the appearance of an electrical field between the aluminum mask and the melt. In order to avoid this electrical field and the metal formation, prior to the ion exchange process an anodization process of the samples was performed [34]. To make an electrical contact the anode plate made from tantalum having an opening in the middle was placed in front of the sample and positioned in a teflon holder. We used a stainless steel plate as a second electrode. The anodization process was carried out in $10 \%(\mathrm{v} / \mathrm{v})$ solution of sulphuric acid (Caledon Laboratories Ltd., Canada) at $0 \mathrm{C}^{\circ}$ with a voltage difference of $15 \mathrm{~V}$ across electrodes [34]. In order to adjust the ion exchange time, various waveguides with different exchange times were fabricated and tested with a solution of deionized water containing $3 \mu \mathrm{m}$ polystyrene microspheres (refractive index 1.59, specific gravity $1.05 \mathrm{~g} / \mathrm{cm}^{3}$, Polysciences Inc., USA). The aluminium was removed in aluminum etchant (Transene Company Inc., USA). $10 \mu \mathrm{m}$ wide waveguides that were exchanged for $17 \mathrm{~min}$ at $360 \mathrm{C}^{\circ}$ yielded the largest propulsion speeds. These carried two modes in width and single mode in depth of the waveguide.

The waveguides were polished at the end phases on a MECAPOL P320 lapping machine (Presi, France) with diamond disks $(220 \mu \mathrm{m}$ and $40 \mu \mathrm{m}$,) and consecutively with diamond suspension $(9 \mu \mathrm{m}, 6 \mu \mathrm{m}$, and $1 \mu \mathrm{m})$. Final polishing was done with a colloidal silica suspension $(0.04 \mu \mathrm{m})$. All polishing materials were purchased from Allied High Tech Products Inc., USA.

Waveguide Surface Functionalization. The surface of the glass samples with the waveguides was either used after thorough cleaning or functionalized via a silanization reaction [35] to form a self-assembled monolayer (SAM) with a functional end group. The functionalization 
with $-\mathrm{NH}_{2}$ was carried out following a procedure suggested by Lee et al. and Toworfe et al. [36, 37]. In order to create a sufficient amount of $-\mathrm{OH}$ groups at the surface of the waveguide sample necessary for the silanization reaction, the ion exchanged waveguide samples were immersed in a mixture of $70 \% \mathrm{H}_{2} \mathrm{SO}_{4}$ and $30 \% \mathrm{H}_{2} \mathrm{O}_{2}$ (v/v) (Caledon Laboratories Ltd., Canada) for $2 \mathrm{~h}$ (piranha solution). The SAM containing an amino group at the end of the silane was formed by immersion of the sample in a 5\% (v/v) mixture of 3-aminopropyltriethxysilane (APTES, Sigma-Aldrich) (5\% APTES, toluene) for $16 \mathrm{~h}$. The formation of the APTES SAM was checked by measuring the contact angle (Rame-Hart Instrument Co., USA). The measured contact angle of the silanized samples was $43.0^{\circ} \pm 3.2^{\circ}$, in excellent agreement with the values reported by Lee et al. of $41.6^{\circ} \pm$ $2.8^{\circ}[36]$ and Toworfe et al. of $42^{\circ}[37]$.

Optical Tweezers Experimental Setup. Linearly polarized light at $\lambda=1064 \mathrm{~nm}$ from an Nd:YAG laser (CrystaLaser IRCL-1.5W-1064, USA) with an output power of $1.5 \mathrm{~W}$ was end-fire coupled into a channel waveguide by a $40 \mathrm{x}$ microscope objective (NA $=0.65$, Newport, USA,). All measurements were performed with TE polarized light.

The waveguide-laser system was mounted independently from the inverted observation microscope (Zeiss, Germany) but in its optical path. The observation microscope was mounted on a heavy duty lateral translation stage to move the objective lens along the waveguide for imaging and for assuring constant coupling efficiency (see below). A scheme of the experimental setup is depicted in Fig.1.

$3 \mu \mathrm{m}$ microspheres (refractive index 1.59 , specific gravity $1.05 \mathrm{~g} / \mathrm{cm}^{3}$, Polysciences Inc., USA) having either amino or carboxyl functional groups on their surface were dispersed in deionised water (Milli-Q). 
A simple cuvette was built on top of the waveguide samples with the help of a doublesided adhesive silicone spacer (500 $\mu \mathrm{m}$ thickness, Sigma-Aldrich, USA) and a cover glass (BK 7) on top. The particle containing solution was filled into the cuvette and left for 10 minutes for particles to settle down on the surface of the waveguide.

The $\mathrm{pH}$ of the water-particle suspension was adjusted by hydrochloric acid (Caledon Laboratories Ltd., Canada) and sodium hydroxide (Caledon Laboratories Ltd., Canada). Since the particles contained functional groups, the adjustment of the $\mathrm{pH}$ was carried out directly in the water particle suspension. The ionic strength of the mixture was controlled by addition of appropriate amounts of sodium chloride (Caledon Laboratories Ltd., Canada).

The propulsion of the particles was recorded $\sim 20 \mathrm{~mm}$ away from the coupling objective by a modified web camera mounted on top of the eyepiece of the inspection microscope. In order to ensure a constant coupling efficiency of the laser beam throughout the entire experimental series (and therefore a constant optical power in the evanescent field), the intensity of the scattered light from the field distribution at the end of the waveguide (opposite to the coupling side) was monitored with a camera. The integrated intensity was obtained by using ImageJ and yielded a variation between individual measurements of less than $3 \%$. The field of view of the objective used to monitor the propulsion of particles was calibrated by an objective micrometer (MA285, Meiji Techno, Japan) and was $140 \mu \mathrm{m}$. The propulsion speed was calculated by using an open source program Tracker (by Douglas Brown, Cabrillo College, USA) that is based on measuring the displacement of a particle in time. The errors in speed calculation arose due to the error in estimating position of the particle and non-constant speed of the particle related to the quality of the functional layer on the waveguide. A single speed data point is the average of 7-10 individual measurements per particle. Typically 3-5 particles had been considered. 


\section{Results and Discussion}

Particles in the Absence of Coulomb Interaction. Initial experiments were carried out with $3 \mu \mathrm{m}$ particles in the absence of charged functionalizations (polystyrene microspheres containing only a slight anionic charge from a sulfate ester, used to achieve hydrophilic particles; particle type: 17134-15, Polysciences Inc., USA). The waveguides were also used without any surface functionalization. Therefore, this particle-waveguide combination did not exhibit any Coulomb interaction. Typical propulsion speeds were $5.06 \pm 0.47 \mu \mathrm{m} / \mathrm{s}$.

The propulsion speed of particles depends on the intensity of the evanescent field that decays away from the surface of the channel waveguide and on the ability of the gradient force to attract particles to the surface. During Coulomb interaction free measurements particles exhibited bouncing motion on the waveguide surface. This demonstrated that particles were not attached to the surface. In order to control the waveguide - particle separation we introduced Coulomb interaction that would enable increase/decrease in the separation distance in the case of repulsive/attractive interaction, respectively, and successively change the propulsion speed due to a different momentum transfer from the evanescent field.

Amino-Amino Group Interaction. Both waveguide and particles were functionalized with $-\mathrm{NH}_{2}$ groups that was supposed to lead to a repulsive, electrostatic Coulomb interaction, if the $\mathrm{pH}$ drives the functionality into the charged, ionic $-\mathrm{NH}_{3}{ }^{+}$state. A scheme of the particle waveguide configuration is shown in Fig.2. The propulsion speed of these amino-particles on the amino-waveguide is presented in Fig.3. For small pH values ( $\mathrm{pH} 4$ - 4.5) no propulsion could be achieved, the particles were stuck and did not even show any Brownian motion. With increasing $\mathrm{pH}$, or decreasing charge density, the propulsion set in, increased steeply to a maximum speed and 
decreased slowly to no propulsion at $\mathrm{pH} 6.15$ and higher $\mathrm{pH}$ values. At high $\mathrm{pH}$ the particles showed only Brownian motion. The maximum speed was found at $\mathrm{pH}=5.15$. Fig. 4 shows a time series of images of a moving particle from right to left. The time interval between two successive images is 2 seconds.

As previously mentioned, in evanescent tweezers the propulsion speed of the particles depends on the strength of the evanescent field and decreases with the movement of a particle away from the surface. Therefore, electrostatic repulsion between the particle and the waveguide surface should increase the distance between both: the higher the charge density on both objects the stronger the repulsion force and the larger the distance. That should result in a decreasing speed with decreasing $\mathrm{pH}$. However, the results contradict this simple explanation. In the experiment we observed that for $\mathrm{pH}$ values around 6 the microspheres are bouncing on the waveguide's surface, and at moments when they are touching the surface there is noticeable light scattering from the evanescent field. However, the optical field is not strong enough to capture and drag the particles toward the surface of the waveguide. By decreasing the $\mathrm{pH}$, some particles are immobilized (that is they are attached to the surface and show neither Brownian motion nor motion due to the evanescent field) in the area above the channel and outside of it. Only those microspheres that are still bouncing can, at some point in time, be moved by the evanescent field. It is worth to notice that the intensity of the scattered light from particles immobilized on the surface of the channel and that from the moving particle is different: it is larger for immobilized particles. As it will be clear from further discussion this difference in intensity is due to the change in the separation distance between particle and waveguide: the closer a particle is located to the surface of the waveguide the larger is the amount of photons scatters from the particle. The fact that some particles were immobilized while the rest still continued to bounce was attributed to 
inhomogeneities in the density of the APTES SAM on the surface of glass sample, leading to roughness and therefore to particle traps [38,39].

For $\mathrm{pH}$ values below 5 all particles are attached to the surface without any evidence of Brownian motion and cannot be moved by the evanescent field. Since an increase in the repulsive force with decreasing $\mathrm{pH}$ could not account for the sticking of the particles to the surface of the glass, we attributed the sticking to an appearance of a dipolar force, originating from negative charge induction on the surface of the glass by the positively charged microparticles or vice versa. Induction of the opposite charge is possible when a charged particle is in proximity of a dielectric surface and repels or attracts electrons toward the surface, depending on the charge of the particle. Similarly, it is possible to induce an opposite charge by charged particle when there is a difference in the charge density between the particle and the considered surface. This effect can only appear when the charge densities on the surface of the particle and the surface of the waveguide are not identical. There are two reasons why we assumed this condition applied. First, we were dealing with a combination of a flat and a curved surface, making the geometry asymmetric, and secondly, the functionalization of the particles and the waveguides were carried out differently leading to a different density of the $-\mathrm{NH}_{2}$ groups. Therefore, an increase in the positive charge density on the surfaces of the particles due to a $\mathrm{pH}$ change induced more negative charge on the surface of glass, thus, increasing attractive forces due to the dipole formation.

The propulsion speed of the microparticles, among other parameters (like e.g. shape of the particle, refractive index of particle and media), depends on the intensity of the evanescent field and the friction force between the particle and the water it is dispersed in [13]. The intensity of the evanescent field is maximal on the surface of the waveguide and exponentially decays away from the surface. Feiler et al. [40] and Marti et al. [41] have studied changes in friction between two 
surfaces carrying charges controllable by the $\mathrm{pH}$. During experiments that employed atomic force microscopy (in the first case a silica sphere was attached to the tip of a cantilever and positioned near a titanium dioxide wafer [40]; and in the second case the cantilever was equipped with silicon nitride tip and interrogated an oxidized silicon surface [41]) authors studied adhesion and friction between two surfaces due to an electrostatic interaction as a function of $\mathrm{pH}$. Marti et al. [41] have concluded that a variation in an electrostatic interaction with changing $\mathrm{pH}$ results in a frictional force change. Feiler et al. [40] attributed variations in friction to changes in the separation distance between particles and substrate. The increase in friction with decreasing separation distance is steep: near the surface of the substrate the frictional force drastically increases from several $\mathrm{nN}$ (for $0.55 \mathrm{~nm}$ separation and above) to about $35 \mathrm{nN}$ (for $0.45 \mathrm{~nm}$ separation). Thus, a change in the separation distance of $0.1 \mathrm{~nm}$ results in an increase of almost one order of magnitude in the friction force. Authors speculated on the content of the layer between particle and wafer surface: whether it is atomic constituents of silicon dioxide and titanium dioxide or a monolayer of bound water attached to surfaces.

Thus, the net force, resulting from electrostatic repulsion due to the presence of positive charges on the amino groups and attraction attributed to the induction of the negative charge on the surface of the samples creating dipoles, drove the microspheres closer to the surface, leading to higher friction and smaller propulsion speed. Reduction in the separation distance between particles and waveguide -in comparison to the uncharged or minimal charged state $(\mathrm{pH}>6.24)$ lead in a pH range of $5.15-5.5$ to an increase in the propulsion speed of the microspheres driven by a particle position at a higher evanescent field. This speed rapidly decreased for $\mathrm{pH}<5$ because of increased friction forces. 
During all experiments at $\mathrm{pH}$ values below 5 it was impossible to start moving particles that were attached to the surface at the beginning of an experiment. This confirmed the presence of high frictional forces at $\mathrm{pH}$ values below 5 .

Since the position of the maximum speed on the $\mathrm{pH}$ scale depends on both electrostatic repulsion and dipole attraction caused by the difference in charge density between particle and waveguide surface, it should be possible to shift the maximum speed position towards the neutral $\mathrm{pH}$ values by changing the charge density on one of surfaces (e.g. on the surface of waveguide by decreasing the silanization time).

Dispersion of positively charged particles in water containing negative $\mathrm{Cl}^{-}$- ions results in electrical double layer formation [42]. Water molecules are polar [43]. The presence of the permanent dipole moment will attract water to a charged surface. In the case of microspheres carrying positively charged amino group, chlorine ions as well as oxygen atoms from water molecules are attracted to the surface of the particles. Propulsion of particles by an evanescent field will therefore also drag an additional layer adjacent to the surface of the particles.

In order to check the role of the increase in the effective size of the particle due to the double layer formation we screened the surface charges by changing the ionic strength: adding sodium chloride into the particle suspension. The $\mathrm{pH}$ dependence of the propulsion speed for 0.1 $\mathrm{mM}$ and $1 \mathrm{mM}$ of $\mathrm{NaCl}$ is presented in Fig.5. Comparing Fig.3 and Fig.5a shows that an addition of $0.1 \mathrm{mM} \mathrm{NaCl}$ does not change the $\mathrm{pH}$ dependence of the propulsion speed. The position of the maximum on the $\mathrm{pH}$ scale and the maximum speed value remained identical within the experimental errors. An increase in the amount of the $\mathrm{NaCl}$ to $1 \mathrm{mM}$ (Fig.5b) increased the maximum speed and shifted the peak position on the $\mathrm{pH}$ scale to a lower $\mathrm{pH}$. Fig.6 compares the speed versus $\mathrm{pH}$ data at very low ionic strength (no $\mathrm{NaCl}$, only few ions due to $\mathrm{pH}$ adjustment) 
and the data at $1 \mathrm{mM} \mathrm{NaCl}$. Presence of negatively charged $\mathrm{Cl}^{-}$ions in the dispersion partially screened charges on both surfaces, reducing particles-waveguide Coulomb interaction. In order to obtain the same net force between particles and the waveguide it was necessary to increase the density of the positive charges on the surfaces by reducing $\mathrm{pH}$. Therefore, the maximum speed was found at lower $\mathrm{pH}$ values.

The theory of double layer considers the formation of several layers of ions carrying counter-charge: the stationary layer (Stern layer) of immobile counter-ions attached to the charged surface; the diffuse layer of counter-ions that still feel the potential of the charged surface, but can move in and from the bulk of the solution; and bulk of the solution, where the influence of the surface potential is negligible [42]. The so-called slipping plane defines the boundary beyond which an exponentially decaying surface potential is close to the potential of the bulk solution. The physical slipping plane separates parts of the solution that move with the charged surface of the particle and the solution that is located beyond the diffused layer. This part of the solution flows around the particle and exhibits therefore a tangential velocity component relative to the surface boundary of the layers. The Debye length defines the thickness of the layer moving with the charged surface (that is the Stern and the diffuse layer) and is inversely proportional to the square root of the ionic strength [42]: increase in the ionic strength of the solution results in a reduction of the Debye length and vice versa.

For solution containing $1 \mathrm{mM}$ of $\mathrm{NaCl}$ the Debye length is on the order of $10 \mathrm{~nm}$. Water dissociation into $\mathrm{H}_{3} \mathrm{O}^{+}$and $\mathrm{OH}^{-}$ions defines the upper limit on the Debye length: for pure water the Debye length cannot exceed $680 \mathrm{~nm}$. In practice, ion impurities present in water result in a Debye length of a few $100 \mathrm{~nm}$ [42]. Thus, a small, in comparison to the size of particles, change in the hydrodynamic radius cannot solely account for an increase in the propulsion speed. 
The propulsion force from the evanescent field depends on the gradient of the dielectric permittivity at the particle-medium interface $[25,44]$. The presence of ions with strong electrostatic fields creates local changes in the orientation of water molecules forming the so-called hydration shells around solvated ions [45]. Bulk solution experiments [46,47] and a theoretical study [48] demonstrate that the dielectric permittivity depends linearly on the salt concentration (for molar salt concentrations up to $1.5 \mathrm{M})[45]$ :

$$
\varepsilon(\mathrm{c})=\epsilon_{0}+\beta \mathrm{c}
$$

where $\varepsilon(c)$ is the dielectric permittivity of the solution with a salt concentration $c$ and, $\varepsilon_{0}$ the dielectric constant of the pure, ion free, solvent. The phenomenological coefficient $\beta$ is negative for most salts and in particular for $\mathrm{NaCl}$, used in present study, varies from $-10.77 \mathrm{M}^{-1}$ to $-12.8 \mathrm{M}^{-}$ ${ }^{1}$ [45]. The introduction of a charged surface and its double layer formation modifies the distribution of the counter- and co-ions, making the dielectric permittivity position dependant [49]:

$$
\epsilon(r)=\epsilon_{0}(c)+4 \pi \alpha_{+} c_{+}(r)+4 \mu \alpha_{-} c_{-}(r)
$$

with $\varepsilon_{0}(\mathrm{c})$ the dielectric permittivity of the bulk solution depending on the concentration of the dissolved salt, and $c_{+}, \alpha_{+}$and $c_{-}, \alpha_{-}$the concentrations and polarizabilities of the positive and negative ions, respectively. For the ions used in the present research $\alpha<0[45,50]$, indicating that the dielectric permeability decreases with increasing ion concentrations. According to Ben-Yaakov et al. [45] this decrease can be as large as half of the bulk value.

Iglič et al. studied the influence of solvated ions on the dielectric permittivity of water near charged surfaces [51]. The authors represent the water molecules as Langevin dipoles and consider changes in dipole orientation and depletion near the surface due to the presence of the solvated ions. It was demonstrated that the effective dielectric permittivity of the solution gradually decreases toward the surface. Das et al. [52] studied the double layer formation near a charged 
surface in the case of a field dependant permittivity due to the presence of ions in the solution. They demonstrate that the dielectric permittivity decreases with approaching the surface. The decrease is a function of the electrical double layer potential gradient: an increase in the electrical double layer potential gradient leads to a decrease of the dielectric permittivity. In the case of 1 $\mathrm{mM} \mathrm{NaCl}$ solution, when the Debye length is smaller in comparison to the salt free solution, the gradient of the electrical double layer potential is higher resulting in lower values of the dielectric permittivity.

Therefore by adding salt to the studied system the dielectric permittivity in the bulk as well as near the surface of the particles changes. In combination with a reduced Debye length, this drop in the dielectric permittivity results in an increase of the dielectric permittivity gradient leading to a larger propulsion force.

This argument of cause also holds for the charged waveguide surface. If the dielectric permittivity drops in front of the waveguide, the electrical field distribution of the propagating waveguide mode is influenced. Because the glass waveguide itself is unchanged, the refractive index step, $\Delta \mathrm{n}$, from the waveguide into the solution is increased in the presence of the higher salt concentration in comparison to the lower salt concentration. This leads to a higher evanescent field directly at the waveguide surface and to a steeper exponential decay of the evanescent field. That means the gradient of the evanescent field is enhanced, and therefore the propulsion force and successively the propulsion speed enlarged.

An additional effect responsible for the propulsion speed enhancement at higher salt concentration might be the interfacial charge regulation [53]: the local $\mathrm{pH}$ close to a charged surface is changed due to the Stern layer and regulates/adapts the surface charge density, both on the particles and on the waveguide. This leads in principle to an additional change in the Debye 
length and therefore, in the case of the particle, in a slight change in the hydrodynamic radius as well into a changes in the local gradients of the dielectric permittivity on both surfaces.

The speed enhancement is a combination of the above discussed enhancement of the propulsion force due to effects of the field dependent dielectric permittivity of the solution near a charged surface and its regulation as well as the local friction. The combination of all these effects will need a thorough theoretical treatment.

Carboxyl-Amino Group Interaction. As above mentioned, the pKa value of the amino group is around 9. By reducing the $\mathrm{pH}$ of a solution below the $\mathrm{pKa}$ value of the amino group, the concentration of the positively charged $-\mathrm{NH}_{3}{ }^{+}$groups exponentially increases. In case of a carboxyl group with a $\mathrm{pKa}$ around 2 , increase in the $\mathrm{pH}$ above 2 exponentially increases the concentration of the negatively charged $-\mathrm{COO}^{-}$groups. Therefore, operating between the $\mathrm{pKa}$ values of the two functional groups simultaneously creates opposite charges. However, an increase in the density of charge of one sign is accompanied by a decrease in the density of the charge carrying the opposite sign [29].

We implemented particles with carboxyl functionalization and a waveguide surface with amino functionalization to achieve an attractive Coulomb interaction. The attractive force between the waveguide and the particles depends on the functionalization density of both surfaces and must have a maximum value at some $\mathrm{pH}$ (depending on both surfaces' functionalization density). Under optimum $\mathrm{pH}$ conditions, when the attraction between particles and waveguide surface is at its maximum it was expected that particles were either immobilized on the surface of the waveguide due to the high friction between the two surfaces or to show a minimum in propulsion speed. A 
$\mathrm{pH}$ change off of the optimum $\mathrm{pH}$, in both directions (larger and smaller) should lead to an increase in the propulsion speed.

The $\mathrm{pH}$ dependence of the propulsion speed of the carboxylated particles on the amino functionalized waveguide is shown on Fig.7. As expected, a range of $\mathrm{pH}$ values was found where propulsion was impossible: for $\mathrm{pH}$ values between 3.7 and 6.5 particles were attached to the waveguide and no motion could be observed. Decreasing the $\mathrm{pH}$ below 3.7 and increasing the $\mathrm{pH}$ above 6.5 reduced the attractive electrostatic forces and propulsion set in. Outside the optimum $\mathrm{pH}$ condition $(3.7<\mathrm{pH}<6.5)$ the decrease in Coulomb attraction decreased the friction forces due to an increased particle-waveguide separation distance. The decrease in the speed both to high and low $\mathrm{pH}$ values was probably caused again by dipole forces due to an induction of opposite charges on the surface of particle or waveguide, respectively. The same argument as discussed above also applied here: an asymmetric geometry and a different functionalization density resulted in different charge densities on the surfaces of particles and waveguide.

The $\mathrm{pH}$ dependence of the propulsion speed investigated at higher ionic strength, at $1 \mathrm{mM}$ $\mathrm{NaCl}$ is presented on Fig.8 (for comparison the data of Fig.7 are included). As in the case of two surfaces with amino groups, the $\mathrm{pH}$ value at which maximum speed is observed was shifted with respect to the investigations without $\mathrm{NaCl}$ due to charge shielding caused by the sodium and chlorine ions, respectively. It is worth noticing that the value of maximum speed at low $\mathrm{pH}$ did not change dramatically with the addition of $\mathrm{NaCl}$, while that one at high $\mathrm{pH}$ increased almost three fold. This is probably due to the fact that at low $\mathrm{pH}$ the charge density on particles is low and the influence of the layer of counter- and coins adjacent to the microspheres was negligible. The opposite situation applied for the speed maximum at high $\mathrm{pH}$. 


\section{Conclusion}

We successfully demonstrated propulsion of amino- and carboxyl-functionalized dielectric microparticles by an optical tweezers of silver ion-exchanged channel waveguides functionalized with amino groups. It was experimentally observed that the speed of both kinds of microspheres in the evanescent field of the functionalized waveguides depended on the $\mathrm{pH}$ of the particle suspension. The dependence of the propulsion speed of microspheres on $\mathrm{pH}$ was explained based on the variation in friction force with the particle-waveguide separation distance. The presence of maximum propulsion speed values with changing $\mathrm{pH}$ was attributed to the appearance of a dipole force caused by the induction of the opposite charge in either microparticle or waveguide due to different charge densities on both surfaces. The influence of the ionic strength of the solution on the propulsion speed of the microparticles was attributed to a slight change in the hydrodynamic radius of the particles, mainly to the reduction of the dielectric permittivity of the solution near the charged surfaces of the microspheres and the waveguide, and to interfacial charge regulation subsequently leading to an increase in the scattering force. Taking these results into account, it should be possible by an appropriate adjustment of the charge density on the waveguide to obtain a minimum or maximum propulsion speed at a suitable $\mathrm{pH}$ for living cells, such as red blood cells.

Acknowledgement. The authors like to thank NSERC and the Government of Ontario in form of the ERA Award for financial help. The Canadian Foundation of Innovation and the Ontario Innovation Trust are thanked for equipment grants. S.M. likes to thank the Government of Canada for the CRC program. David Shoesmith's Lab in the Department of Chemistry at Western is thanked for help and advice in polishing. The Western Nanofabrication Facility is thanked for 
access in order to fabricate the waveguide devices. Hao Jiang and Erden Ertorer are thanked for helpful advice in channel waveguide fabrication. 


\section{Figure captions}

Fig.1 Schematic diagram of the experimental setup: a - waveguide coupling objective, b imaging objective, $\mathrm{c}$ - waveguide substrate, A - objective position of coupling efficiency alignment and $\mathrm{B}-$ objective position for imaging.

Fig.2 Schematic diagram of microsphere - waveguide configuration both carrying amino groups at $\mathrm{pH}$ values below the $\mathrm{pKa}$ of the amino group without $\mathrm{NaCl}$ (very low ionic strength).

Fig.3 Propulsion speed of microspheres versus $\mathrm{pH}$ in the case of an amino-amino group interaction. The error bars at the data points showing zero propulsion speed have been omitted, as no movement and therefore no data analysis was performed.

Fig.4 Time series of images showing the propulsion of an $\mathrm{NH}_{2}$-functionalized particle on a $\mathrm{NH}_{2}$ -functionalized waveguide from right to left at maximum speed $(\mathrm{pH}=5.15)$. The images were recorded with $2 \mathrm{~s}$ interval and without an IR filter.

Fig.5 Propulsion speed of microspheres versus $\mathrm{pH}$ in case of amino-amino group interaction in a) $0.1 \mathrm{mM} \mathrm{NaCl}$ solution, and b) $1 \mathrm{mM} \mathrm{NaCl}$ solution. The error bars at the data points showing zero propulsion speed have been omitted, as no movement and therefore no data analysis was performed. In the cases where no error bar appear despite the zero speed data points, the error is within the symbol size. 
Fig.6 Propulsion speed versus $\mathrm{pH}$ at very low ionic strength and at $1 \mathrm{mM}$ of $\mathrm{NaCl}$. The error bars at the data points showing zero propulsion speed have been omitted, as no movement and therefore no data analysis was performed. In the case where no error bar appear despite the zero speed data points, the error is within the symbol size.

Fig.7 $\mathrm{pH}$ dependence of the propulsion speed of carboxylated microspheres on amino functionalized waveguide. The error bars at the data points showing zero propulsion speed have been omitted, as no movement and therefore no data analysis was performed.

Fig.8 pH dependence of the propulsion speed of carboxyl functionalized microspheres near an amino functionalized waveguide with and without $1 \mathrm{~m} \mathrm{M} \mathrm{NaCl}$. The error bars at the data points showing zero propulsion speed have been omitted, as no movement and therefore no data analysis was performed. In the cases where no error bar appear despite the zero speed data points, the error is within the symbol size. 


\section{References}

1. Wolff A.; Perch-Nielsen I.R.; Larsen U.D.; Friis P.; Goranovic G.; Poulsen C.R.; Kutter J.P.; Telleman P. Lab Chip 2003, 3, 22.

2. Andersson H.; Van Der Berg A. Sens. Actuators B 2003, 92, 315.

3. Ashkin A. Phys. Rev. Lett. 1970, 24, 156.

4. Grier D.G. Nature 2003, 424, 810.

5. Curtis J.E.; Koss B.A.; Grier D.G. Opt. Commun. 2002, 207, 169.

6. Rice S.E.; Purcell T.J.; Spudich J.A. Methods Enzymol. 2003, 361, 112.

7. Ke P.C.; Gu M. Appl. Opt. 1999, 38, 160.

8. Ghislain L.P.; Switz N.A.; Webb W.W. Rev. Sci. Instr. 1994, 65, 2762.

9. Keyser U.F.; Koeleman B.N.; van Dorp S.; Krapf D.; Smeets R.M.M.; Lemay S.G.; Dekker N.H.; Dekker C. Nature Phys. 2006, 2, 473.

10. Wright G.; Tucker M.J.; Morton P.C.; Sweitzer-Yoder C.L.; Smith S.E. Curr. Opin. Obstet. Gyn. 1998, 10, 221.

11. Curtis J.E.; Grier D.G. Opt. Lett. 2003, 28, 872.

12. Wang G.M.; Sevick E.M.; Mittag E.; Searles D.J.; Evans D.J. Phys. Rev. Lett. 2002, 89, 050601.

13. Erickson. D.; Serey X.; Chen Y.-F.; Mandal S. Lab. Chip 2011, 11, 995.

14. Kawata S.; Sugiura T. Opt. Lett. 1992, 17, 772.

15. Kawata S.; Tani T. Opt. Lett. 1996, 21, 1768.

16. Ng L.N.; Luff B.J.; Zervas M.N.; Wilkinson J.S. Appl.Phys. Lett. 2000, 76, 1993.

17. Ng L.N.; Luff B.J.; Zervas M.N.; Wilkinson J.S. Opt. Commun. 2002, 208, 117.

18. Gaugiran S.; Gétin S.; Fedeli J.M.; Derouard J. Opt. Express 2007, 15, 8146. 
19. Grujic K.; Helleso O.G.; Wilkinson J.S.; Hole J.P. Optics Communications 2004, 239, 227.

20. Ahluwalia B.S.; Hellesø O.G.; Subramanian A.Z.; Chen J.; Wilkinson J.S.; Chen X. Proc. SPIE 2010, 7613, 76130R.

21. Grujic K.; Helleso O.G. Optics Express 2007, 15, 6470.

22. Grujic K.; Helleso O.G.; Hole J.P.; Wilkinson J.S. Optics Express 2005, 13, 1.

23. Lin S.; Crozier K.B. Proc. SPIE 2011, 7950, 79500W-1.

24. Néel D.; Gétin S.; Ferret P.; Rosina M.; Fedeli J.M.; Hellesø O.G. Appl. Phys. Lett. 2009, 94, 253115.

25. Gaugiran S.; Gétin S.; Fedeli J.M.; Colas G.; Fuchs A.; Chatelain F.; Dérouard J. Opt. Express 2005, 13, 6956.

26. George J.N.; Weed R.I.; Reed C.F. J. Cell. Physiol. 1971, 77, 51.

27. Sagvolden G.; Giaever I.; Pettersen E.O.; Feder J. Proc. Nat. Acad. Sci. U.S.A. 1999, 96, 471.

28. Ahluwalia B.S.; McCourt P.; Huser T.; Helleso O.G. Optics Express 2010, 18, 21053.

29. CRC Handbook of Chemistry and Physics. Cleveland, Ohio : CRC Press, 2011-2012, v.92.

30. Introduction to Glass Integrated Optics. S. Iraj Najafi, Artech House, 1992.

31. Jackel J.L.; Vogel E.M.; Aitchinson J.S. Appl. Optics 1990, 29, 3126.

32. Integrierte Optik. Karthe, Müller, Akademische Verlagsgesellschaft, Leipzig, 1991.

33. Walker R.G.; Wilkinson C.D.W.; Wilkinson J.A.H. Appl. Optics 1983, 22, 1923.

34. The surface treatment and finishing of aluminium and its alloys. Forth edition, v.1, by Wernick S. and Pinner R., Robert Draper Ltd., Teddington, 1972.

35. An Introduction to Ultrathin Organic Films: From Langmuir-Blodgett to Self-Assembly. Ulman A., Academic Press, Boston, Toronto.

36. Lee M.H.; Brass D.A.; Morris R.; Composto R.J.; Ducheyne P. Biomaterials 2005, 26, 1721. 
37. Toworfe G.K.; Composto R.J.; Shapiro I.M.; Ducheyne P. Biomaterials 2006, 27, 631.

38. Creager S.E.; Hockett L.A.; Rowe G.K. Langmuir 1992, 8, 854.

39. Hallab N.J.; Bundy K.J.; O’Connor K.; Moses R.L.; Jacobs J.J. Tissue Engineering 2001, 7, 55.

40 Feiler A.; Larson I.; Jenkins P.;Attard P. Langmuir 2000, 16, 10269.

41. Marti A.; Hahner G.; Spencer N.D. Langmuir 1995, 11, 4632.

42. Physics and Chemistry of Interfaces. Butt H.-J., Graf K., Kappl M., Wiley-VCH, 2003.

43. Atkins' Physical Chemistry Atkins P, de Paula J., Oxford, New York: Oxford University Press, 2006.

44. Brevik I. Phys. Rep. 1979, 52, 133.

45. Ben- Yaakov D.; Andelman D.; Podgornik R. J. Chem. Phys. 2011, 134, 074705.

46. Glueckauf E. Trans. Faraday Soc. 1964, 60, 1637.

47. Wei Y.; Sridhar S. J. Chem. Phys. 1992, 96, 4569.

48. Chandra A. J. Chem. Phys. 2000, 113, 903.

49. Hatlo M.M.; van Roij A.; Lue L. Europhysics Letters, 2012, 97, 28010.

50. Hasted J.B.; Ritson D.M.; Collie C.H. J. Chem. Phys. 1948, 16, 1.

51. Iglič A.; Gongadze E.; Bohinc K. Bioelectrochemistry 2010, 79, 223.

52. Das S.; Chakraborty S.; Mitra S.K. Phys. Rev. E 2012, 85, 051508.

53. Hartvig R.A.; van de Weert M.; Østergaard J.; Østergaard 1.; Jensen H. Langmuir 2011, 27, 2634. 
FIG.1

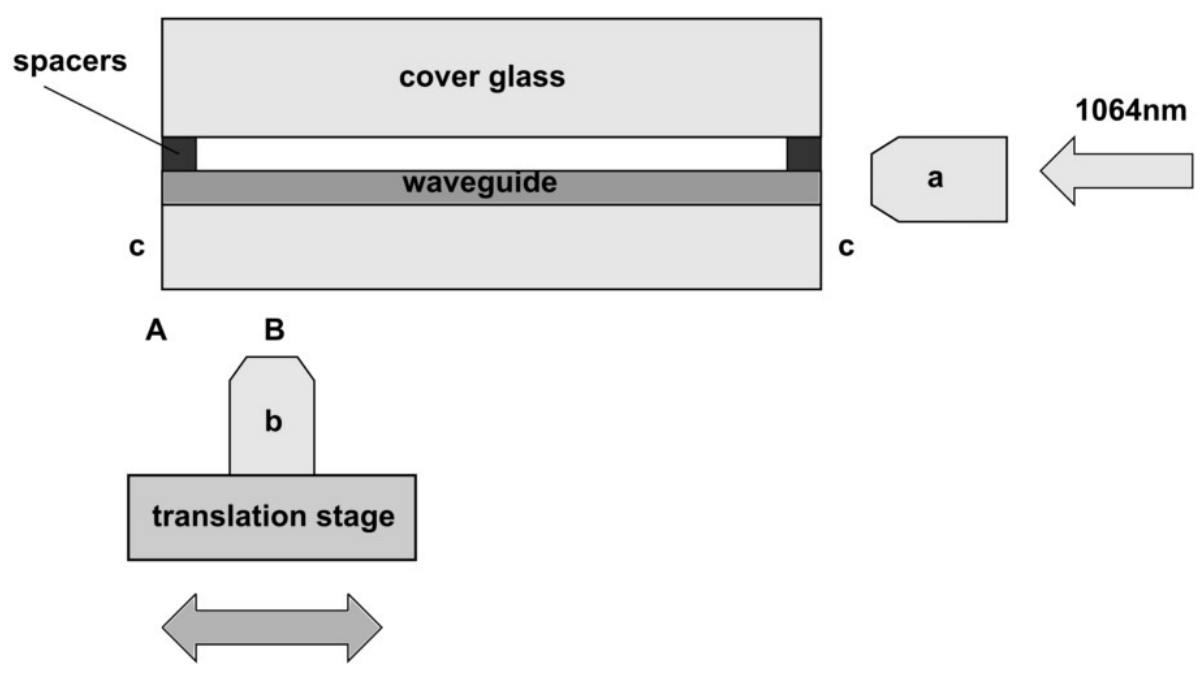

FIG.2
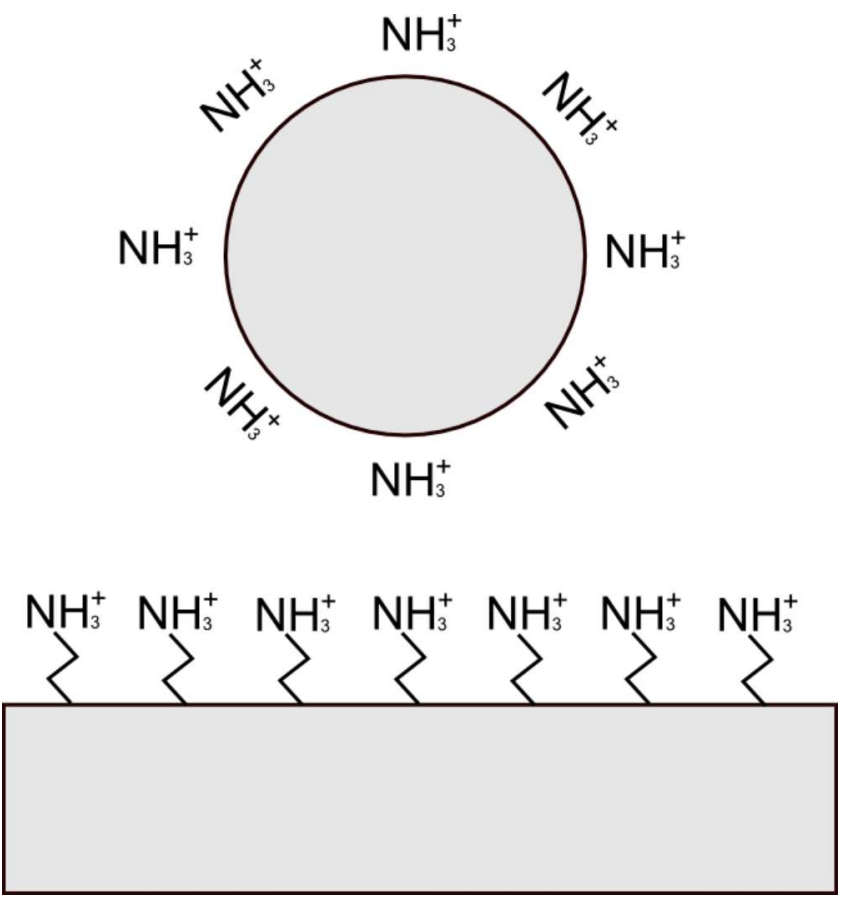
FIG.3

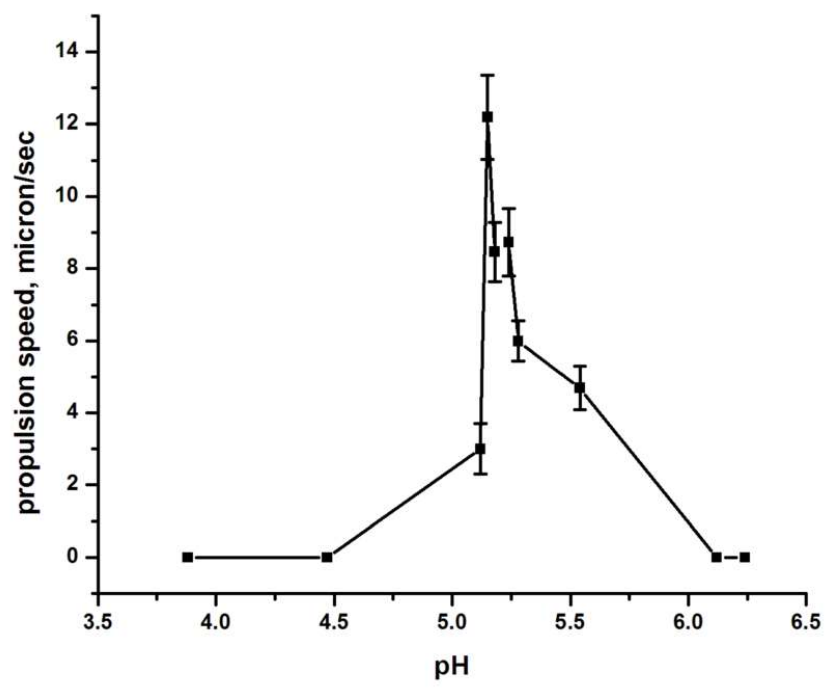

FIG.4

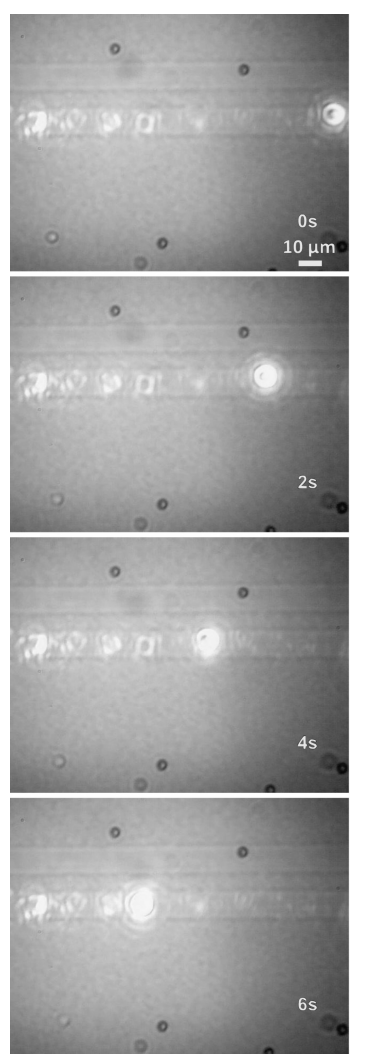


FIG.5a

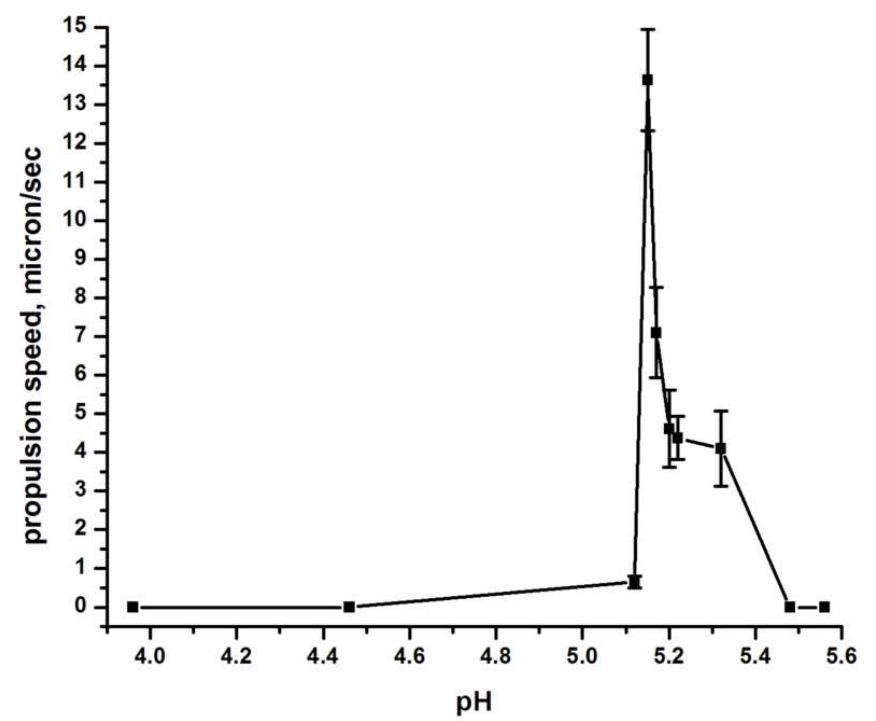

FIG.5b

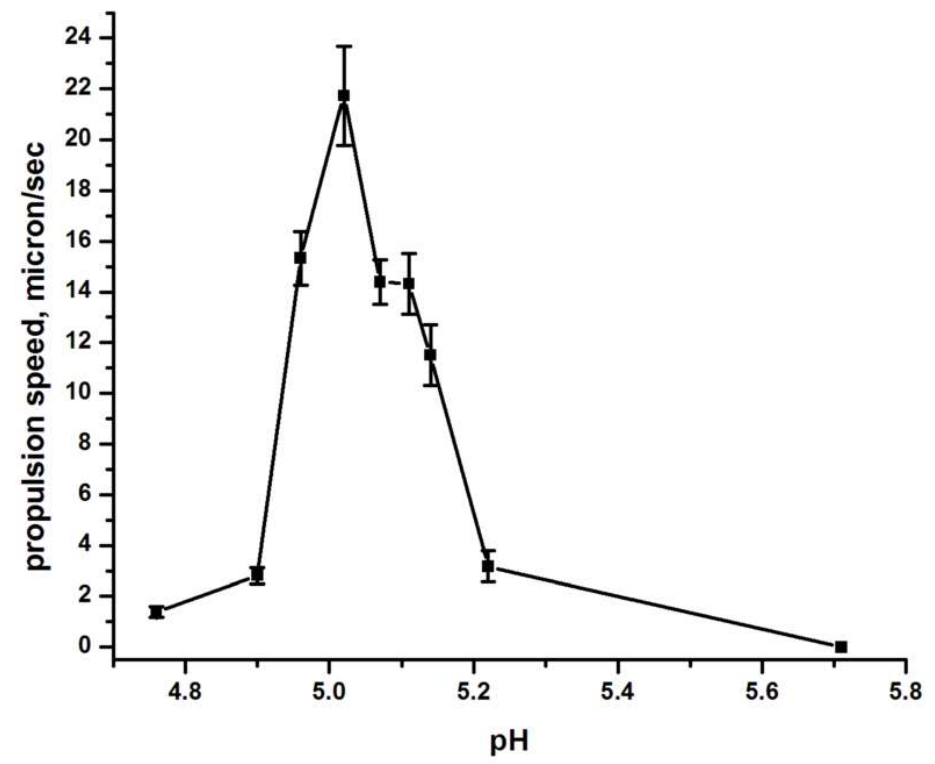


FIG.6

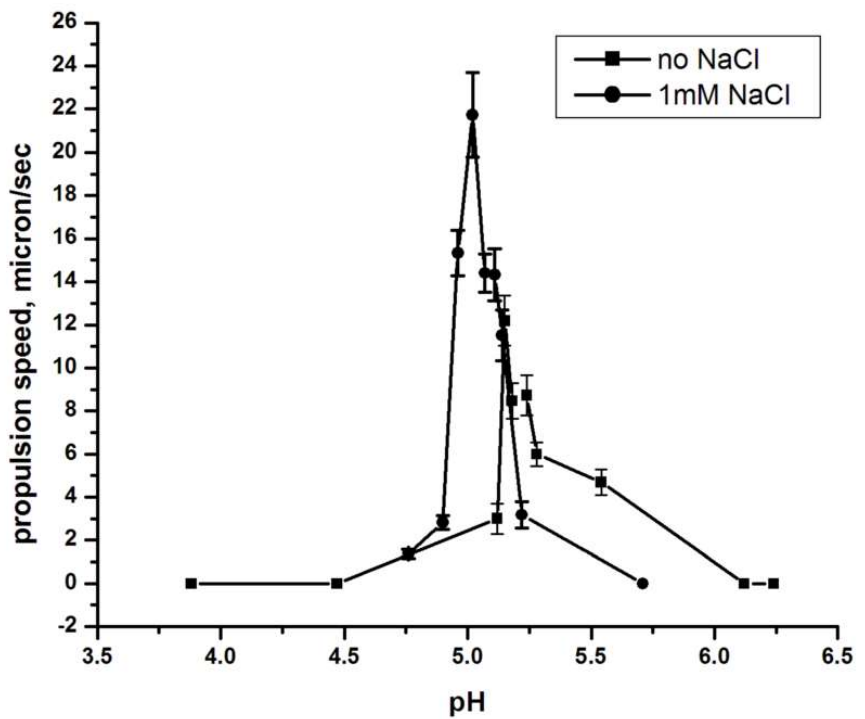

FIG.7

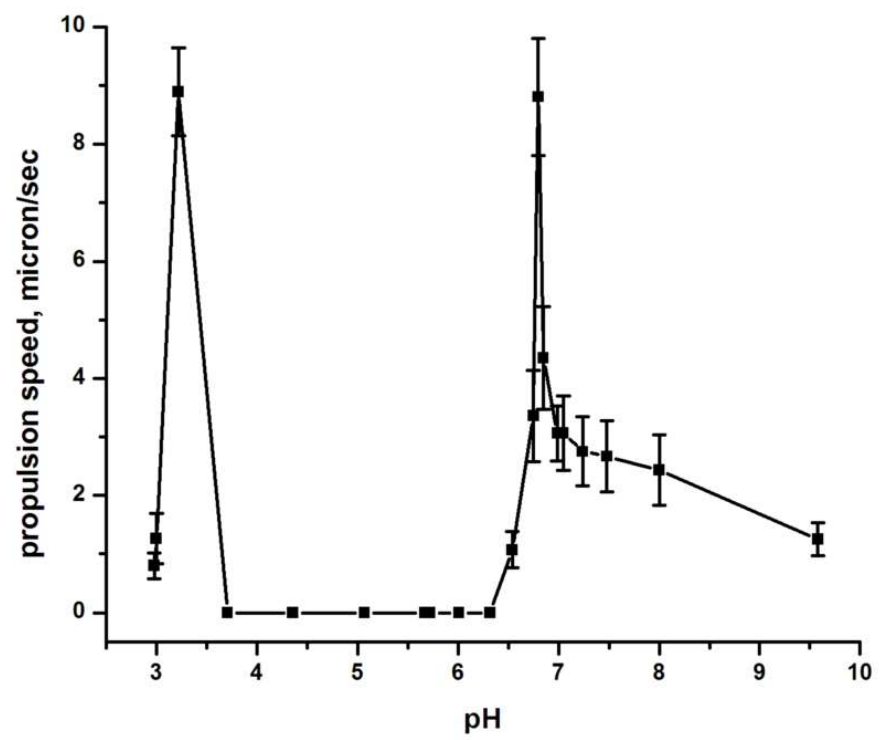


FIG. 8

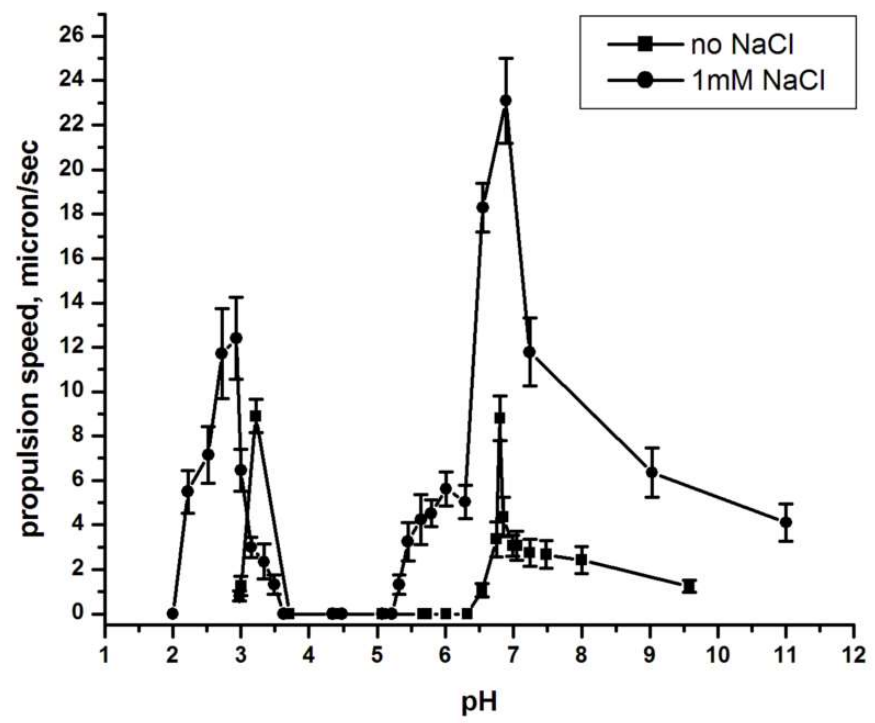

